\title{
Transient SH Wave Propagation of Elastic Plate
}

\author{
Kotaro Miura ${ }^{1 *}$, Makoto Sakamoto ${ }^{2}$, and Yuji Tanabe ${ }^{3}$ \\ ${ }^{1}$ Seikei University, Department of Systems Design Engineering, 3-3-1 Kichijojikitamachi, \\ Musashino-shi, Tokyo 180-8633, Japan \\ ${ }^{2}$ Niigata University School of Medicine, Department of Health Sciences, 2-746 Asahimachi, Chuo- \\ ku, Niigata 951-8518, Japan \\ ${ }^{3}$ Niigata University, Department of Mechanical Engineering, 2-8050 Ikarashi, Nishi-ku, Niigata 950- \\ 2181, Japan
}

\begin{abstract}
We consider the transient wave propagation problem of linear, isotropic and elastic plate applied SH impact loading on the surface. Analytical solution of half-space obtained by the inverse Fourier-Laplace double transform using Cagniard-De Hoop method. The wave propagation problem of plate was considered by using a half-space exact solution and reflect wave from the boundary of plate are expressed using the image method. Some numerical results of stress and displacement components are presented. The mathematical technique appear in the basic problem can apply to the transient $\mathrm{P}$ wave propagation and more advanced problems.
\end{abstract}

\section{Introduction}

The analytical research of the wave propagation for the elastic body was studied by Lamb [1] firstly who consider the problem applied the normal line loading on the surface of elastic half space. The exact solution of this problem was given by Cagniard [2] and developed by De Hoop [3]. Their mathematical technique of the inverse Fourier-Laplace double transform is known as Cagniard-De Hoop method. Miklowitz [4] studied the transient problem the elastic rods and plates. In this paper, the transient problem of isotropic and elastic plate applied SH impact loading on the surface. The SH wave propagation is appropriate to consider the basic problem of elastic plate because the SH wave yields reflected SH wave only at the boundary. Thus, the complication of the mode conversion of $\mathrm{P}$ and SV waves can be removed. An analytical solution for half-space was obtained by the inverse Fourier-Laplace transform using Cagniard-De Hoop method. It is necessary to treat properly the multivalued function of the square root of a complex number in the inverse Laplace transform. However, there is no literature that concludes clearly for the handling. This study describes the mathematical technique for calculating the multivalued function for the numerical results. Furthermore, the wave propagation problem of plate was considered by applying the analytical solution of half-space and the image method which expressing the reflect wave from the boundary of plate. The mathematical technique for handling the multivalued function of the square root of a complex number in the problem can apply to the $\mathrm{P}$ wave propagation and more advanced problems.

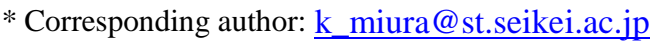




\section{Formulation of the problem}

Consider the anti-plane problem of half-space applied SH impact loading suddenly. The wave equation can be written as follows [5]:

$$
\begin{aligned}
& \frac{\partial^{2} w}{\partial x^{2}}+\frac{\partial^{2} w}{\partial y^{2}}=\frac{1}{c^{2}} \frac{\partial^{2} w}{\partial t^{2}}, \\
& c=\sqrt{\frac{\mu}{\rho}}
\end{aligned}
$$

where $w, c, \mu$ and $\rho$ represent displacement component of $z$-direction, phase velocity of SH wave, shear modulus and density of elastic solid, respectively.

Boundary and initial condition can be described by:

$$
\begin{aligned}
& \left.\tau_{y z}\right|_{y=0}=Q \delta(x) H(t), \\
& \left.w\right|_{t=0}=\left.\dot{w}\right|_{t=0}=0,
\end{aligned}
$$

where $Q, \delta(x)$ and $H(t)$ represent the dimensionless magnitude of the load, Dirac delta function and Heaviside unit step function, respectively.

Fourier and Laplace transform are defined following equations:

$$
\begin{gathered}
\left\{\begin{array}{c}
\mathscr{F}[f(x)] \equiv f^{F}(\xi)=\int_{-\infty}^{\infty} f(x) e^{-i s \xi x} \mathrm{~d} x, \\
\mathcal{F}^{-1}\left[f^{F}(\xi)\right] \equiv f(x)=\frac{s}{2 \pi} \int_{-\infty}^{\infty} f^{F}(\xi) e^{i s \xi x} \mathrm{~d} \xi,
\end{array}\right. \\
\left\{\begin{array}{c}
\mathcal{L}[f(t)] \equiv f^{L}(s)=\int_{0}^{\infty} f(t) e^{-s t} \mathrm{~d} t, \\
\mathcal{L}^{-1}\left[f^{L}(s)\right] \equiv f(t)=\frac{1}{2 \pi i} \int_{\mathrm{Br}} f^{L}(s) e^{s t} \mathrm{~d} s .
\end{array}\right.
\end{gathered}
$$

Fourier and Laplace transform applied to the Eqs. (1), (3) and (4). The transform solution of displacement and stress can be obtained as follows $\left(\operatorname{Re}[s]>0, \operatorname{Re}\left[\sqrt{\xi^{2}+v^{2}}\right]>0\right)$ :

$$
\begin{aligned}
w^{F L} & =-\frac{Q}{s^{2} \mu \sqrt{\xi^{2}+v^{2}}} \exp \left[-s \sqrt{\xi^{2}+v^{2}} y\right], \\
\tau_{y z}^{F L} & =\frac{Q}{s} \exp \left[-s \sqrt{\xi^{2}+v^{2}} y\right], \\
\tau_{x z}^{F L} & =-\frac{i Q \xi}{s \sqrt{\xi^{2}+v^{2}}} \exp \left[-s \sqrt{\xi^{2}+v^{2}} y\right],
\end{aligned}
$$

where,

$$
v=1 / c^{2} .
$$

The inverse Fourier transform of Eq. (7) can be written as 


$$
\begin{aligned}
w^{L} & =-\frac{Q}{2 \pi} \int_{-\infty}^{\infty} \frac{1}{s \mu \sqrt{\xi^{2}+v^{2}}} \exp \left[-s\left(\sqrt{\xi^{2}+v^{2}} y-i \xi x\right)\right] \mathrm{d} \xi, \\
\tau_{y z}^{L} & =\frac{Q}{2 \pi} \int_{-\infty}^{\infty} \exp \left[-s\left(\sqrt{\xi^{2}+v^{2}} y-i \xi x\right)\right] \mathrm{d} \xi \\
\tau_{x z}^{L} & =-\frac{i Q}{2 \pi} \int_{-\infty}^{\infty} \frac{\xi}{\sqrt{\xi^{2}+v^{2}}} \exp \left[-s\left(\sqrt{\xi^{2}+v^{2}} y-i \xi x\right)\right] \mathrm{d} \xi .
\end{aligned}
$$

The inverse Laplace transform can be applied using Cagniard-De Hoop method. The idea of the Cagniard-De Hoop method is to deform the path of integration in the $\xi$-plane in such a manner that the inverse Laplace transform of the integration along the new path of integration can be obtained by inspection, analogously to the following elementary property of the one-sided Laplace transform [6]:

$$
\mathcal{L}^{-1}\left[\int_{t_{1}}^{\infty} e^{-s t} f(t) d t\right] \equiv f(t) H\left(t-t_{1}\right)
$$

The desired path of integration in the $\xi$-plane is obviously defined by the following equation:

$$
t=\sqrt{\xi^{2}+v^{2}} y-i \xi x
$$

The Eq. (11) can be solved for complex variable $\xi$ to yield

$$
\xi_{ \pm}=\frac{i x t \pm y \sqrt{t^{2}-v^{2} R^{2}}}{R^{2}}
$$

where,

$$
R=\sqrt{x^{2}+y^{2}}
$$

In the $\xi$-plane, Eq. (12) represents a hyperbola as shown in Fig. 1. The vertex of the hyperbola is $i x v / R$ located on the between the branch points at $\pm i v$. Thus, a branch cut along the imaginary axis, where is represented by hatching in Fig. 1, make $\sqrt{\xi^{2}+v^{2}}$ a single-valued function. The original contour $\Gamma$ and Cagniard-de Hoop contour $\Gamma_{1}$ are equivalent because there are no poles between $\Gamma$ and $\Gamma_{1}$.

By substituting Eq. (11) to Eq. (9), the deformed Eq. (9) are the elementary form of Laplace transform. Thus, the inverse Laplace transform of Eq. (9) can be expressed as follows:

$$
\begin{aligned}
w & =-\frac{Q}{\mu} \int_{v R}^{t}\left(\frac{1}{\sqrt{\xi_{+}^{2}(\tau)+v^{2}}} \frac{\partial \xi_{+}(\tau)}{\partial \tau}-\frac{1}{\sqrt{\xi_{-}^{2}(\tau)+v^{2}}} \frac{\partial \xi_{-}(\tau)}{\partial \tau}\right) \mathrm{d} \tau, \\
\tau_{y z} & =\frac{Q}{2 \pi} H(t-v R) \cdot \frac{2 y t}{R^{2} \sqrt{t^{2}-v^{2} R^{2}}}, \\
\tau_{x z} & =\frac{Q}{2 \pi} H(t-v R) \cdot \operatorname{Im}\left[\frac{\xi_{+}(t)}{\sqrt{\xi_{+}^{2}(t)+v^{2}}} \frac{\partial \xi_{+}(t)}{\partial t}-\frac{\xi_{-}(t)}{\sqrt{\xi_{-}^{2}(t)+v^{2}}} \frac{\partial \xi_{-}(t)}{\partial t}\right],
\end{aligned}
$$


where,

$$
\begin{aligned}
& \frac{\partial \xi_{+}}{\partial t}=\frac{i x+y t / \sqrt{t^{2}-v^{2} R^{2}}}{R^{2}}, \\
& \frac{\partial \xi_{-}}{\partial t}=\frac{i x-y t / \sqrt{t^{2}-v^{2} R^{2}}}{R^{2}} .
\end{aligned}
$$

Since $\sqrt{\xi^{2}+v^{2}}$ in Eq. (14) is a multivalued function, it needs to be careful calculating in the practice. The mathematical technique described below can treat the multivalued value of $\sqrt{\xi^{2}+v^{2}}$ accurately.

The function of $\sqrt{\xi^{2}+v^{2}}$ is expressed in polar form as follows:

$$
\sqrt{\xi^{2}+v^{2}}=\sqrt{(\xi+i v)(\xi-i v)}=\sqrt{r_{12} e^{i \theta_{12}} \cdot r_{11} e^{i \theta_{11}}},
$$

where,

$$
\begin{aligned}
& r_{12} e^{i \theta_{12}}=(\xi+i v), \\
& r_{11} e^{i \theta_{11}}=(\xi-i v) .
\end{aligned}
$$

We consider the point $P$ on the contour $\Gamma_{1}$, and define the argument of Eq. (17) as shown in Fig. 1. The amplitudes of the polar form are written as following equations:

$$
\begin{aligned}
& r_{12}=\sqrt{\operatorname{Re}[\xi]^{2}+(\operatorname{Im}[\xi]+v)^{2}}, \\
& r_{11}=\sqrt{\operatorname{Re}[\xi]^{2}+(\operatorname{Im}[\xi]-v)^{2}} .
\end{aligned}
$$

The arguments of Eq. (17) can be calculated according to the position of point $P$ as follows $(x>0)$ :

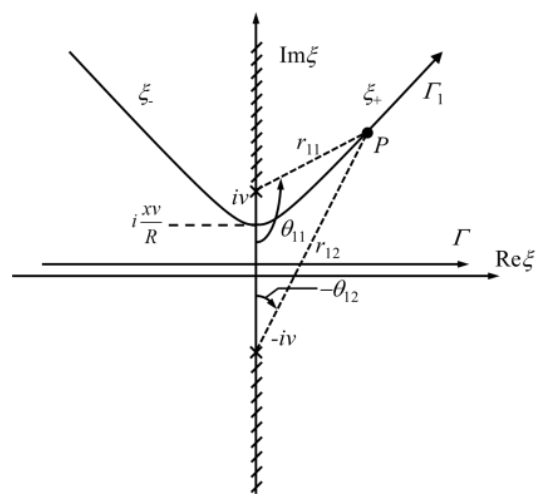

Fig. 1. Cagniard-de Hoop contour $\Gamma_{1}$ and the argument of point $P$ on the contour. 
(i) $\operatorname{Re}[\xi]=0$

$\left\{\begin{array}{l}\theta_{12}=0, \\ \text { if } \operatorname{Im}[\xi]-v>0 \rightarrow \theta_{11}=\pi, \text { (It dose not matter for SH wave problem.) } \\ \text { if } \operatorname{Im}[\xi]-v<0 \rightarrow \theta_{11}=0 .\end{array}\right.$

(ii) $\operatorname{Re}[\xi]<0$

$\left\{\begin{array}{l}\theta_{12}=\frac{\pi}{2}-\tan ^{-1}\left(\frac{|\operatorname{Im}[\xi]|+v}{|\operatorname{Re}[\xi]|}\right), \\ \theta_{11}=-\left\{\frac{\pi}{2}+\tan ^{-1}\left(\frac{|\operatorname{Im}[\xi]|-v}{|\operatorname{Re}[\xi]|}\right)\right\} .\end{array}\right.$

(iii) $\operatorname{Re}[\xi]>0$

$\left\{\begin{array}{l}\theta_{12}=-\left\{\frac{\pi}{2}-\tan ^{-1}\left(\frac{|\operatorname{Im}[\xi]|+v}{|\operatorname{Re}[\xi]|}\right)\right\}, \\ \theta_{11}=\frac{\pi}{2}+\tan ^{-1}\left(\frac{|\operatorname{Im}[\xi]|-v}{|\operatorname{Re}[\xi]|}\right) .\end{array}\right.$

It can be calculated in the same manner when $x<0$. Furthermore, this technique can also be applied to the $\mathrm{P}$ wave propagation problem.

The reflect wave from boundary of plate are SH wave only for the problem in this study. Thus, the reflect wave are expressed by the image method. Image method is a simple method expressing the reflected wave from the plate boundary by folding back the solution of halfspace at the boundary of plate.

\section{Numerical results}

In this study, some numerical results of wave propagation obtained by Eq. (14) are presented when the thickness of plate, $t h X=0.3$. The numerical results show that normalized stress and displacement components are divided by $Q$ and $Q / \mu$, respectively.

Figure 2, 3 and 4 show the contours of stress components $\tau_{y z}, \tau_{x z}$ and displacement component $w$ of SH wave propagation at some normalized times, $t^{*}=0.3,0.6$ and 1.0, respectively. $t^{*}=1.0$ means the wave front approached the position $(X=0.0, Y=1.0)$ on the normalized $X Y$-coordinate $(v=1.0)$. The stress on the wave front replaced with a large value and loading point (surface of plate) is slightly shifted since it is impossible to calculate. The amplitude of reflected $\tau_{y z}$ from the free boundary of plate is opposite in sign because $\tau_{y z}$ vanishes at free surface, whereas $\tau_{x z}$ and $w$ reflected in phase with the incident wave.

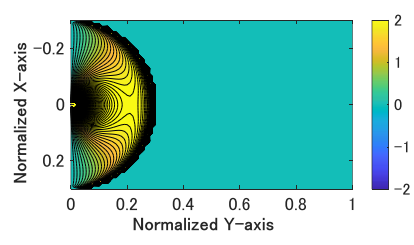

(a) $t^{*}=0.3$

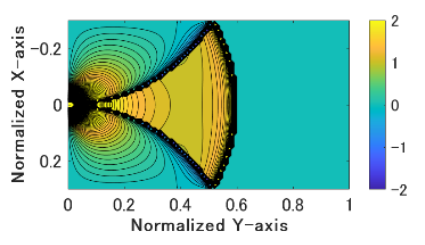

(b) $t^{*}=0.6$

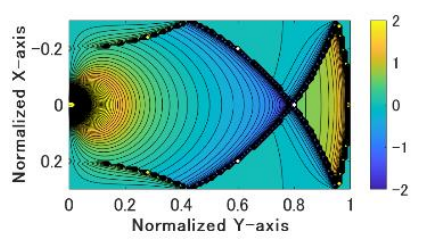

(c) $t^{*}=1.0$

Fig. 2. $\tau_{y z}$ contours of wave propagation in plate (thickness of plate, $t h X=0.3$ ). 


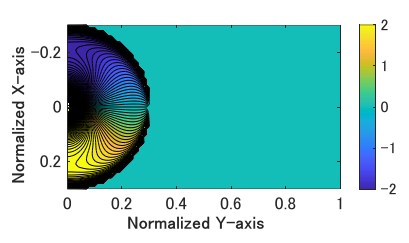

(a) $t^{*}=0.3$

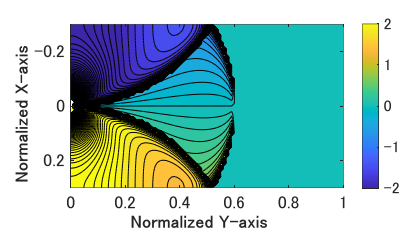

(b) $t^{*}=0.6$

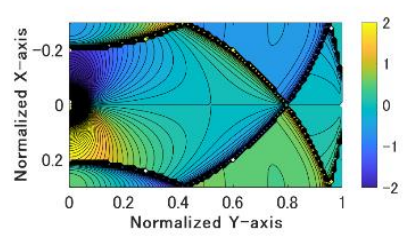

(c) $t^{*}=1.0$

Fig. 3. $\tau_{x z}$ contours of wave propagation in plate (thickness of plate, $t h X=0.3$ ).

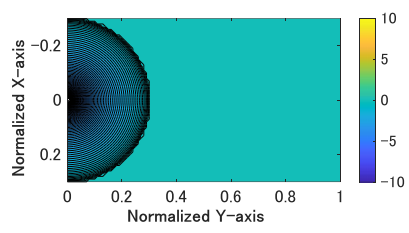

(a) $t^{*}=0.3$

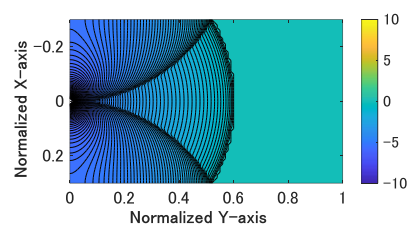

(b) $t^{*}=0.6$

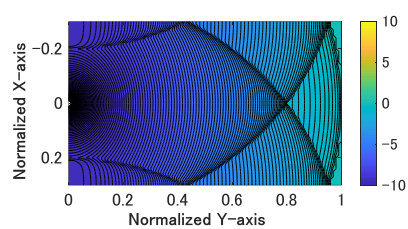

(c) $t^{*}=1.0$

Fig. 4. $w$ contours of wave propagation in plate (thickness of plate, $t h X=0.3$ ).

\section{Conclusion}

We considered the transient wave propagation of plate applied SH impact loading. Firstly, an analytical solution for linear, isotropic and elastic half-space was obtained by the inverse Fourier-Laplace double transform using Cagniard De-Hoop method. It is noted that multivalued functions must be handled carefully in the inverse Laplace transform. Secondary, the wave propagation problem of elastic plate was considered. The reflect waves from boundary of plate were expressed by the image method which a simple method folding back the solution of the half-space at the boundary of plate. The mathematical technique for handling the multivalued function of the square root of a complex number in the problems can apply to the $\mathrm{P}$ wave propagation and more advanced problems.

\section{References}

1. H. Lamb, Phil. Trans. Roy. Soc. (London). Ser. A, 203, pp. 359-371 (1904)

2. L. Cagniard, Réflexion et réfraction des ondes seismiques progressives (GauthiersVillars, Paris, 1939)

3. A. T. De Hoop, Appl. Sci. Res. Sect. B, 8, 1 (1960)

4. J. Miklowitz, The theory of elastic waves and waveguides (North-Holland, New York, 1978)

5. K. F. Graff, Wave motion in elastic solids (Dover Publications, New York, 1991)

6. J. D. Achenbach, Wave propagation in elastic solids (North-Holland, New York, 1980) 